\title{
COMPORTAMIENTO DE LAS PROPIEDADES FÍSICAS Y MECÁNICAS DE CONCRETOS SUSTENTABLES BASE AGREGADOS RECICLADOS Y CENIZA DE BAGAZO DE CAÑA DE AZÚCAR
}

\author{
A. LANDA-SANCHEZ ${ }^{1}$, C. GAONA-TIBURCIO ${ }^{2}$, F. ALMERAYA-CALDERÓN ${ }^{2}$, \\ A. C. RAMÍREZ-GARCÍA ${ }^{3}$, S. MÁRQUEZ-MONTERO ${ }^{4}$, M. A. BALTAZAR-ZAMORA ${ }^{4}$ \\ ${ }^{1}$ Universidad Veracruzana, Doctorado en Ingeniería, FIME, Xalapa, Veracruz, México \\ ${ }^{2}$ Universidad Autónoma de Nuevo León. FIME - CIIIA. Av. Universidad S/N Ciudad Universitaria. \\ San Nicolás de los Garza, Nuevo León, México \\ ${ }^{3}$ Universidad Veracruzana, Facultad de Ingeniería - Ixtaczoquitlán, Orizaba, Veracruz, México \\ ${ }^{4}$ Universidad Veracruzana, Facultad de Ingeniería Civil - Xalapa, Circ. G. Aguirre Beltrán S/N, \\ Lomas del Estadio, CP 91000, Xalapa, Veracruz, México
}

\section{RESUMEN}

En el presente trabajo se evalúan las propiedades físicas y mecánicas de concretos sustentables elaborados a base de sustitución en diferentes \% del Agregado Grueso por Agregados Reciclados, así como la sustitución del $20 \%$ de Cemento Portland por CBCA. El diseño de las mezclas de concreto fue acorde al ACI 211.1 con curado de 28 días. Los ensayos del concreto en estado fresco y endurecido fueron conforme normativa ONNCCE y ASTM. Se determinó revenimiento, temperatura, resistencia a la compresión (F'C), módulo de elasticidad y tracción indirecta, a 14 y 28 días. Los resultados presentan una disminución en la resistencia a la compresión en los concretos sustentables con agregados reciclados y CBCA, relacionada al \% de absorción presente en los AR y la disminución de la adherencia por la pasta presente en ellos.

Palabras clave: CBCA; Agregados reciclados; Concreto sustentables.

\begin{abstract}
In the present work, the physical and mechanical properties of sustainable concrete manufactured based on substitution in different\% of the Coarse Aggregate by Recycled Aggregates are evaluated, as well as the replacement of $20 \%$ of Portland Cement by CBCA. The design of the concrete mixtures was in accordance with the ACI 211.1 with 28 days' curing. The tests of the concrete in fresh and hardened state were in accordance with ONNCCE and ASTM regulations. Slump, temperature, compressive strength $\left(\mathrm{F}^{\prime} \mathrm{C}\right)$, modulus of elasticity and indirect traction were determined at 14 and 28 days. The results show a decrease in the compressive strength in the sustainable concretes with recycled aggregates and CBCA, related to the $\%$ of absorption present in the RA and the decrease of the adherence by the paste present in them.
\end{abstract}

Keywords: CBCA; Recycled aggregates; Sustainable concrete.

\section{RESUMO}

No presente trabalho, são avaliadas as propriedades físicas e mecânicas de concretos sustentáveis fabricados com base na substituição em diferentes\% do Agregado Grosseiro por Agregados Reciclados, bem como a substituição de $20 \%$ de Cimento Portland por CBCA. O projeto das misturas de concreto estava de acordo com o ACI 211.1 com 28 dias de cura. Os testes do concreto em estado fresco e endurecido estavam de acordo com os regulamentos ONNCCE e ASTM. Queda, temperatura, resistência à compressão $\left(\mathrm{F}^{\prime} \mathrm{C}\right)$, módulo de elasticidade e tração indireta foram determinados aos $14 \mathrm{e}$ 28 dias. Os resultados mostram uma diminuição na resistência à compressão nos concretos sustentáveis 
com agregados reciclados e CBCA, relacionados à\% de absorção presente na $\mathrm{AR}$ e à diminuição da aderência pela pasta presente neles.

Palavras-chave: CBCA; Agregados reciclados; Concreto sustentável.

\section{INTRODUCCIÓN}

El tema del reciclaje de concreto se considera muy importante en el intento general un desarrollo sostenible en nuestros tiempos. De forma paralela, está directamente relacionada con (a) el aumento de estructuras de demolición fuera del tiempo de ejecución, (b) la demanda de nuevas estructuras y (c) los resultados de destrucción por fenómenos naturales (terremotos, etc.). La protección del medio ambiente es un factor básico, que está directamente relacionado con la supervivencia de la raza humana. Parámetros como la conciencia ambiental, la protección de los recursos naturales, el desarrollo sostenible desempeña un papel importante en los modernos requerimientos de obras de construcción. Los materiales de construcción son muy significativos en nuestras vidas, porque pasamos el $90 \%$ de nuestro tiempo en edificios o infraestructuras (carreteras, autopistas, puentes, etc.). El sector de materiales de construcción corresponde de 3 - 4\% del total del producto en Europa, y la industria de la construcción, así como obras de construcción, ocupa millones de personas. La necesidad de producción y uso de RCA (Henderson, 1998., BRITE/EURAM, 2002., BRITE/EURAM, 2000., RILEM, 1996., USA-DT, 1997., USA-DT-2000) es hoy en día muy urgente. Este tema ya se ha considerado como muy importante en Europa y en países Desarrollados como E.U.A, Canadá, entre otros. Existe un establecimiento de propuestas para una protección sustancial de las fuentes naturales en el mundo, desafortunadamente no son ni infinitas ni económicas. Se produce volúmenes considerados de desechos de concreto hidráulico, los cuales terminan ilegalmente en áreas no controladas para su depósito. Se sigue el objetivo del desarrollo sostenible y, en consecuencia, se establece la base de una convivencia amistosa entre el hombre y la naturaleza. Datos indican que el 10\% de los agregados usados en Gran Bretaña (Collins, 1996) son RCA, 78.000 toneladas de RCA se utilizaron en Holanda (De Vries, 1996) en 1994, ya que la organización nacional correspondiente admitió que el uso del 20\% de RCA grueso no diferenciaba propiedades del concreto en estado fresco o endurecido. Además, el reciclaje de BDW en el 40\% se ha establecido como objetivo en Alemania (Acker,1996) desde 1991. Un informe reciente de la administración federal de carreteras se refiere a la relativa experiencia de los datos europeos sobre el pavimento de concreto y asfalto reciclado. El rápido desarrollo de la investigación sobre el uso de RCA para la producción de concreto nuevo también ha llevado a la producción de concreto de alto rendimiento y resistencia (Limbachiya, 2000). Debe observarse, por supuesto, que normalmente se recomienda el uso de RCA grueso (hasta un 30\%), pero a menudo se considera necesario añadir super plastificantes (Zankler, 1999., RILEM, 1994) para lograr la trabajabilidad requerida del nuevo concreto. Por estas razones es importante y necesario que la construcción tenga cambios que beneficien y ayuden al mejoramiento de nuestro entorno.

Requiriendo varios elementos como: maximizar la durabilidad del concreto, la conservación de materiales, el uso de residuos y materiales de cementación suplementarios y reciclaje de materiales, como cenizas volantes, escoria de alto horno, humo de sílice, cenizas de cáscara de arroz, ceniza de bagazo de caña y metacaolín, pueden utilizarse como reemplazos parciales del cemento portland, lo que permitiría una reducción a nivel mundial en las emisiones de $\mathrm{CO} 2$ que son de entre el 5 y $7 \%$ del total a nivel mundial producidas por la industria cementera ((Santiago, G. 2016; Landa A.E. et.al., 2018; Baltazar M. et.al., 2016; Santiago, G. 2016), además estos materiales pueden mejorar la durabilidad del concreto [M.L. Berndt 2009].

Una tendencia actual es la utilización del uso de agregado obtenido del concreto triturado, de construcción o demolición, siendo un ejemplo de reciclaje y conservación de materias primas. Aunado al desarrollo sostenible se tiene la Ceniza de bagazo de caña de azúcar (CBCA) que es un residuo 
resultante de la combustión del bagazo en las calderas de los ingenios y se ha demostrado su elevado contenido de sílice ( $\mathrm{SiO} 2)$ y alúmina (Al2O3), teniendo una buena actividad puzolánica como sustituto parcial del cemento portland. Además, siendo un desecho abundante en el Estado de Veracruz, desde esta perspectiva se tendría la utilización de otro residuo para reciclarlo y ayudar al ambiente (Landa A. E. 2018, García D.L 2019, Landa 2019, García D.L 2019, Ruíz 2019, Figueroa 2019). En la presente investigación se utilizaron RCA y CBCA como agregado y sustitución parcial al concreto (Landa 2018; Farid 2015; O. Ojeda 2018), para evaluar las propiedades de este, tanto físicas como mecánicas, y valorar el comportamiento frente a un concreto elaborado con agregado $100 \%$ natural y CPC sin ninguna adición.

\section{METODOLOGÍA EXPERIMENTAL}

\subsection{Acopio y trituración del agregado reciclado.}

El acopio del agregado fue en la calle Enríquez de la Ciudad de Xalapa Veracruz. Se habló con el encargado de la obra y se llevó un camión de $7 \mathrm{~m}^{3}$ de capacidad, se levantó el material con una máquina Excavadora 320 con martillo, se cargó el material y de llevo a la trituradora para gravas de 3/8" a finos. Se depositó el material, en cuanto se descargó se procedió con un marro a disminuir las dimensiones a un tamaño de aproximadamente de 10 a 15 pulgadas para que pudiera pasar por la máquina de trituración. Posterior a esto con un cepillo de acero se eliminó lo más posible los residuos de la base del concreto que se demolió. Se ingresó el material a la máquina y se trituró.

\subsection{Obtención y muestreo de la ceniza de bagazo de caña de azúcar.}

Se obtiene la ceniza de la zafra 2017, muestreada en calderas 2 y 3 del ingenio. Esta caldera es una Babcock Wilcox con capacidad nominal de 3,248 caballos caldera. El horno es un tipo Ward que suministra aire caliente lateralmente y por debajo mediante una placa con rejillas. El muestreo se llevó a cabo durante la mañana aproximadamente a la segunda hora de limpieza de los ceniceros, concentrando la ceniza retirada en un recipiente de material adecuado para resistir la alta temperatura y con el equipo de protección que permitiera realizar el muestreo.

\subsection{Obtención de los agregados naturales.}

Los agregados naturales fueron obtenidos de bancos de material para la construcción, se eligió un banco cerca de la Cd. De Xalapa y éste se encuentra en la lista de bancos de material de la Secretaria de Comunicaciones y Transportes. Para el caso de la grava se solicitó material de $3 / 4$ a finos, y se indicó que se utilizaría para elaboración de concreto para utilizar en pavimento. En el caso de la grava, fue grava de río por las características del mismo banco y ésta fue cribada para que cumpliera con su tamaño nominal para ser caracterizada como una arena.

\subsection{Caracterización de las propiedades físicas de la arena y de la grava.}

Obtenido el material para la elaboración de los concretos se tomaron muestras de cada material para que de acuerdo con la normativa y se caracterizaran de acuerdo a sus propiedades físicas.

Para el caso de la grava, se obtuvo una muestra de aproximadamente $7 \mathrm{~kg}$, por el método de cuarteo, y para el caso de la arena la muestra fue de casi $2 \mathrm{~kg}$ a ambos materiales se les determinó el peso volumétrico seco suelto, el peso volumétrico compacto, su densidad aparente y su porcentaje de absorción. Además de que ambos materiales se procedieron a cribar para su caracterización, todo esto en base a las normas que se muestran en la tabla 1. 
Tabla 1.- Pruebas a agregados.

\begin{tabular}{|c|c|}
\hline Norma & Significado \\
\hline NMX-C-030ONNCCE-2004 & Muestreo de agregados \\
\hline NMX-C-164-ONNCCE-2014 & Determinación de la densidad y absorción agregado grueso \\
\hline NMX-C-165-ONNCCE-2014 & Determinación de la densidad y absorción agregado fino \\
\hline NMX-C-166-ONNCCE-2006 & Determinación del contenido de humedad \\
\hline NMX-C-170-1997-ONNCCE & Reducción de muestras \\
\hline NMXC-073-ONNCCE-2004 & Determinación de la Masa volumétrica \\
\hline NMX-C 077-1997-ONNCCE & Análisis granulométrico \\
\hline NMX-C-416-ONNCCE-2003 & Determinación de Equivalente de arena \\
\hline
\end{tabular}

\subsection{Nomenclatura de los especímenes.}

La nomenclatura será de acuerdo con cada porcentaje utilizado de agregado reciclado, como se muestra en la tabla 2.

Tabla 2.- Nomenclatura de los especímenes de prueba.

\begin{tabular}{|c|c|c|}
\hline REL. a/c & Nomenclatura & Significado \\
\hline \multirow{5}{*}{0.65} & MC & Concreto Normal (100\% CPC y 100\% AN) \\
\cline { 2 - 3 } & M20 & (CONCRETO: 20\% AR -20\% CBCA- 80\% CPC) \\
\cline { 2 - 3 } & M40 & (CONCRETO: 40\% AR -20\% CBCA- 80\% CPC) \\
\cline { 2 - 3 } & M60 & (CONCRETO: 60\% AR -20\% CBCA- 80\% CPC) \\
\cline { 2 - 3 } & M80 & (CONCRETO: 80\% AR -20\% CBCA- 80\% CPC) \\
\cline { 2 - 3 } & M100 & (CONCRETO: 100\% AR -20\% CBCA- 80\% CPC) \\
\hline
\end{tabular}

Dónde:

CPC: Cemento Portland compuesto $30 \mathrm{R}$.

CBCA: Ceniza de bagazo de caña de azúcar.

AR: Agregado reciclado.

AN: Agregado natural.

\subsection{Diseño de mezclas de concreto.}

El método utilizado para el diseño de las mezclas de concreto es de acuerdo a lo que especifica el Instituto Americano del Concreto (American Concrete Institute) en el apartado 211.1, el cual tiene como objetivo determinar la combinación más práctica de los materiales con los que se dispone para producir un concreto que satisfaga los requisitos de comportamiento bajo las condiciones particulares de uso. Para este trabajo el concreto deberá de poseer las siguientes propiedades: en el concreto fresco, trabajabilidad aceptable: en el concreto endurecido, resistencia, durabilidad, densidad, apariencia y por último economía. Las especificaciones que se tomaron para este trabajo son las que se presentan a continuación:

- Concreto Normal.

- Concreto sin aire incluido.

- Relación agua/cemento de 0.65

- Tamaño Máximo Nominal de $19 \mathrm{~mm}$.

- Revenimiento de $7.5 \mathrm{~cm}$. 


\subsection{Ensayos en el concreto fresco.}

Los ensayos realizados en las mezclas de concretos sustentables en su estado fresco, contribuyen en la evaluación de las propiedades de trabajabilidad, temperatura, masa volumétrica y revenimiento. La tabla 3 muestra bajo que norma se realizaron.

Tabla 3.- Pruebas al concreto fresco.

\begin{tabular}{|c|c|}
\hline \multicolumn{1}{|c|}{ Ensayo } & Norma \\
\hline $\begin{array}{c}\text { Muestreo } \\
\text { fresco }\end{array}$ & NMX-C-161-ONNCCE-2013 \\
\hline $\begin{array}{c}\text { Determinación de la temperatura del concreto } \\
\text { fresco }\end{array}$ & NMX-C-435-ONNCCE-2010 \\
\hline $\begin{array}{c}\text { Determinación del revenimiento en el concreto } \\
\text { rendimiento y contenido de aire del concreto } \\
\text { fresco por el método gravimétrico }\end{array}$ & NMX-C-156-ONNCCE-2010 \\
\hline Elaboración y curado de especímenes de ensayo & NMX-C-159-ONNCCE-2016 \\
\hline
\end{tabular}

\subsection{Ensayos al concreto endurecido.}

Los ensayos de concreto endurecido que se analizaron en este proyecto son los que se presentan en la tabla 4.

Tabla 4.- Pruebas al concreto endurecido.

\begin{tabular}{|c|c|}
\hline Ensayo & Norma \\
\hline $\begin{array}{l}\text { Determinación de la resistencia a la } \\
\text { compresión simple de especímenes. }\end{array}$ & NMX-C-083-ONNCCE-2014 \\
\hline $\begin{array}{l}\text { Determinación del módulo de elasticidad } \\
\text { estático y relación de piosson }\end{array}$ & NMX-C-156-ONNCCE-2010 \\
\hline
\end{tabular}

\section{RESULTADOS Y ANÁLISIS.}

\subsection{Propiedades físicas de la arena y de la grava.}

\subsubsection{Masa volumétrica.}

Los resultados del ensaye de masa volumétrica del agregado fino y grueso, en estado suelto y varillado se muestran en la tabla 5:

Tabla 5.- Masa volumétrica de agregados.

\begin{tabular}{|c|c|c|}
\hline Agregado & Norma & Resultado \\
\hline \multirow[b]{2}{*}{ Fino } & \multirow[b]{2}{*}{$\begin{array}{c}\text { NMX-C-073-ONNCCE- } \\
2004\end{array}$} & Masa Volumétrica Suelta: $1,442 \mathrm{Kg} / \mathrm{m} 3$ \\
\hline & & $\begin{array}{l}\text { Masa Volumétrica Varillada: 1,695 } \\
\mathrm{Kg} / \mathrm{m} 3\end{array}$ \\
\hline \multirow[b]{2}{*}{ Grueso } & \multirow{2}{*}{$\begin{array}{c}\text { NMX-C-073-ONNCCE- } \\
2004\end{array}$} & Masa Volumétrica Suelta: $1,332 \mathrm{Kg} / \mathrm{m} 3$ \\
\hline & & $\begin{array}{l}\text { Masa Volumétrica Varillada: 1,433 } \\
\qquad \mathrm{Kg} / \mathrm{m}\end{array}$ \\
\hline
\end{tabular}




\subsubsection{Determinación de la densidad relativa y absorción de agua del agregado fino y grueso.} Los resultados de la densidad relativa y absorción de agua del agregado fino y grueso se presentan en la tabla 6.

Tabla 6.-Densidad y porcentaje de absorción de agregados.

\begin{tabular}{|c|l|c|}
\hline Agregado & Norma & Resultado \\
\cline { 1 - 1 } \multirow{2}{*}{ Fino } & \multirow{3}{*}{ NMX-C-165-ONNCCE-2014 } & $\begin{array}{c}\% \mathrm{~A}=1.80 \% \text { Dr SSS }= \\
\end{array}$ \\
\cline { 1 - 1 } Grueso & & $\begin{array}{c}\% \mathrm{~A}=1.70 \% \text { Dr SSS }= \\
2.60\end{array}$ \\
\hline
\end{tabular}

Como se puede apreciar en la tabla 6, la densidad del agregado pétreo es de 2.20, de acuerdo con el tipo de material, se encuentra dentro de los parámetros de densidades de agregados finos de río. La absorción que se obtiene es de $1.80 \%$. De acuerdo con lo anterior se considera aceptable los resultados obtenidos en este ensaye, para ambos agregados.

\subsection{Concreto fresco.}

\subsubsection{Temperatura.}

En la gráfica 1 se muestra la temperatura de las mezclas de referencia encontrándose dentro de las especificaciones de la norma C-435.

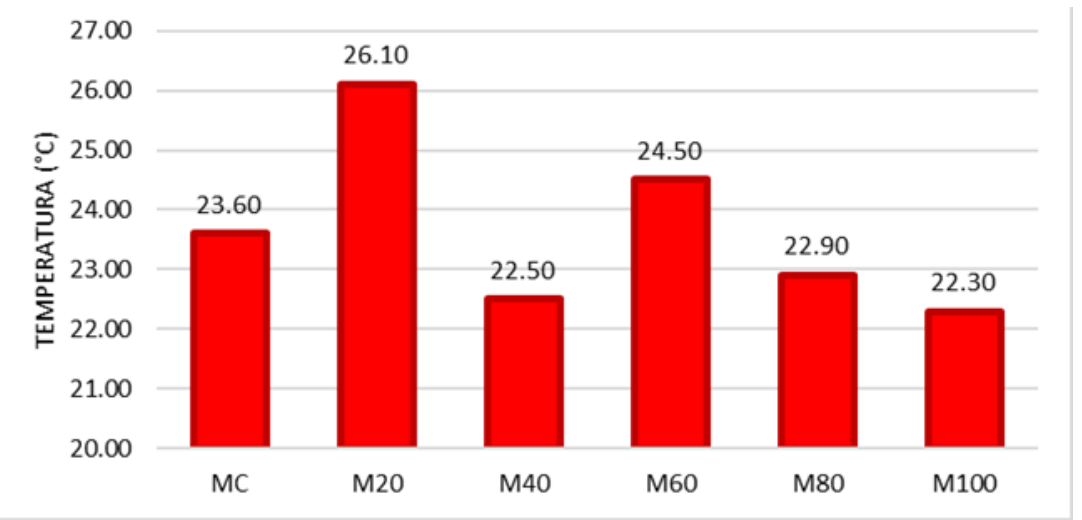

Gráfica 1.- Temperatura de los concretos sustentables en estado fresco.

\subsubsection{Revenimiento.}

Las especificaciones del revenimiento del concreto hidráulico de igual manera están establecida por la norma mexicana NMX-C-155, la cual en su apartado 5.2.2 nos especifica los parámetros que se pueden utilizar en las diferentes circunstancias en las que nos encontremos. La especificación que rige para la evaluación de los resultados de revenimiento es del valor nominal del cual fue diseño, el cual es de 7.5 $\mathrm{cm}$, por lo que tenemos una tolerancia de $\pm 2.5 \mathrm{~cm}$. En la gráfica 2 se aprecia que la mayoría de los resultados de los revenimientos están fuera de la especificación de la norma, pero dentro de la misma hay notas aclaratorias que indican que si los revenimientos son inferiores al límite especificado, el concreto puede aceptarse si no existen dificultades de colocación (ONNCCE, 2014). 


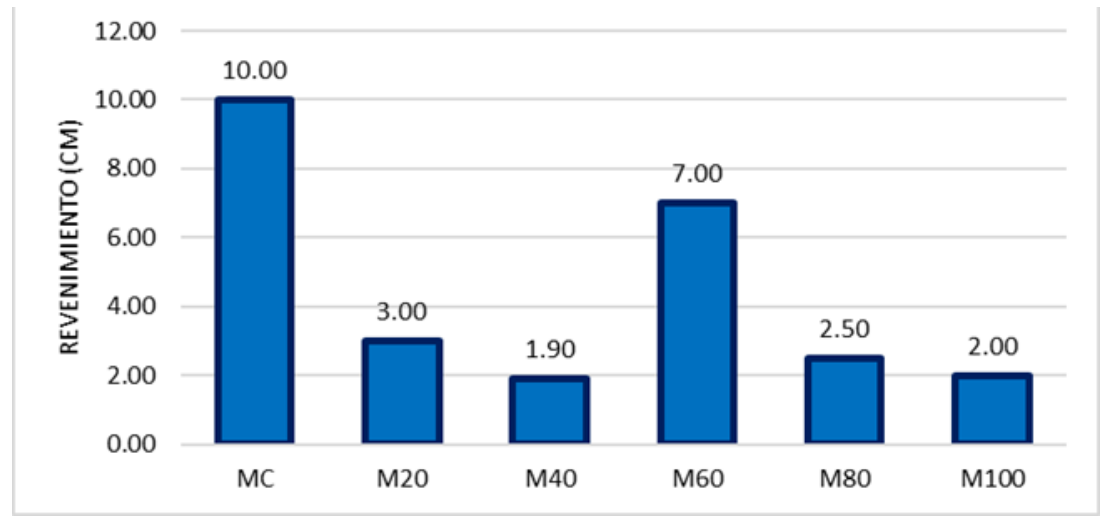

Gráfica 2.-Revenimiento de los concretos sustentables.

Los revenimientos son aceptables puesto que solo se elaboraron probetas de ensaye. Los resultados obtenidos de revenimientos muestran un decrecimiento de la trabajabilidad del concreto considerablemente y esto se atribuye al porcentaje utilizado de puzolanas y al porcentaje de absorción que tiene el agregado reciclado.

\subsubsection{Masa Unitaria.}

En este apartado se presentan los resultados de la masa unitaria de los concretos sustentables elaborados. Como se especifica en la norma mexicana NMX-C155, los concretos hidráulicos de uso estructural deben de tener una masa unitaria normal en estado fresco de 1,900 kg/m3 hasta 2,400 Kg/m3 (ONNCCE, 2014). Como se muestra en la gráfica 3 las seis mezclas se encuentran dentro del rango de aceptación. Siendo la mínima la M100, que es la mezcla con 100\% de agregado reciclado.

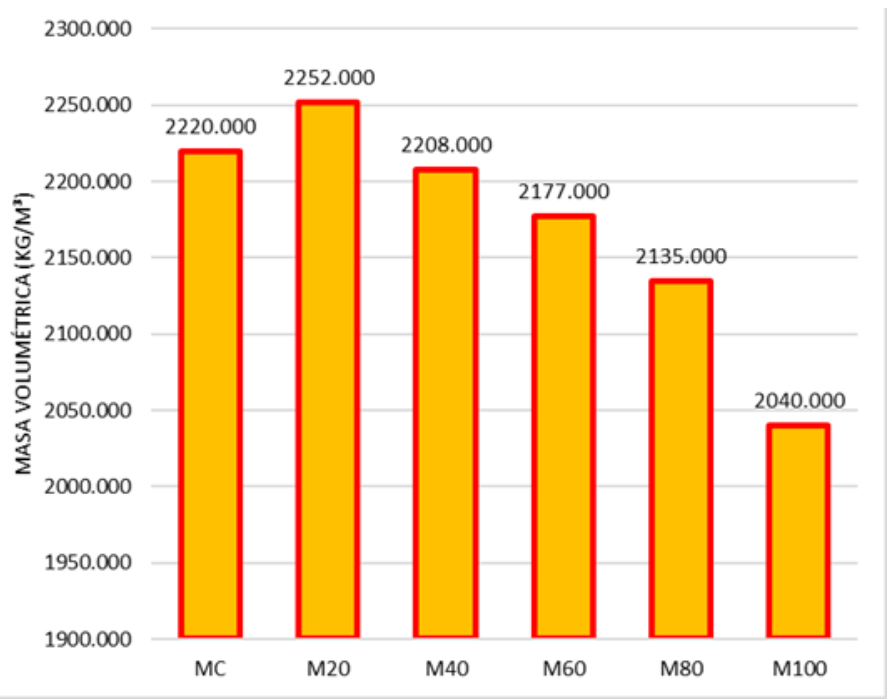

Gráfica 3.-Masa unitaria de los concretos sustentables.

\subsection{Concreto endurecido.}

\subsubsection{Resistencia a la compresión.}

En el gráfico 4 se muestra la resistencia a la compresión de las 6 mezclas a las edades de 14 y 28 días. De acuerdo con el diseño de la mezcla el concreto debe alcanzar resistencias dentro de los parámetros de $180 \mathrm{~kg} / \mathrm{cm} 2$ a $200 \mathrm{~kg} / \mathrm{cm} 2$ que fue de acuerdo a la relación agua cemento. 


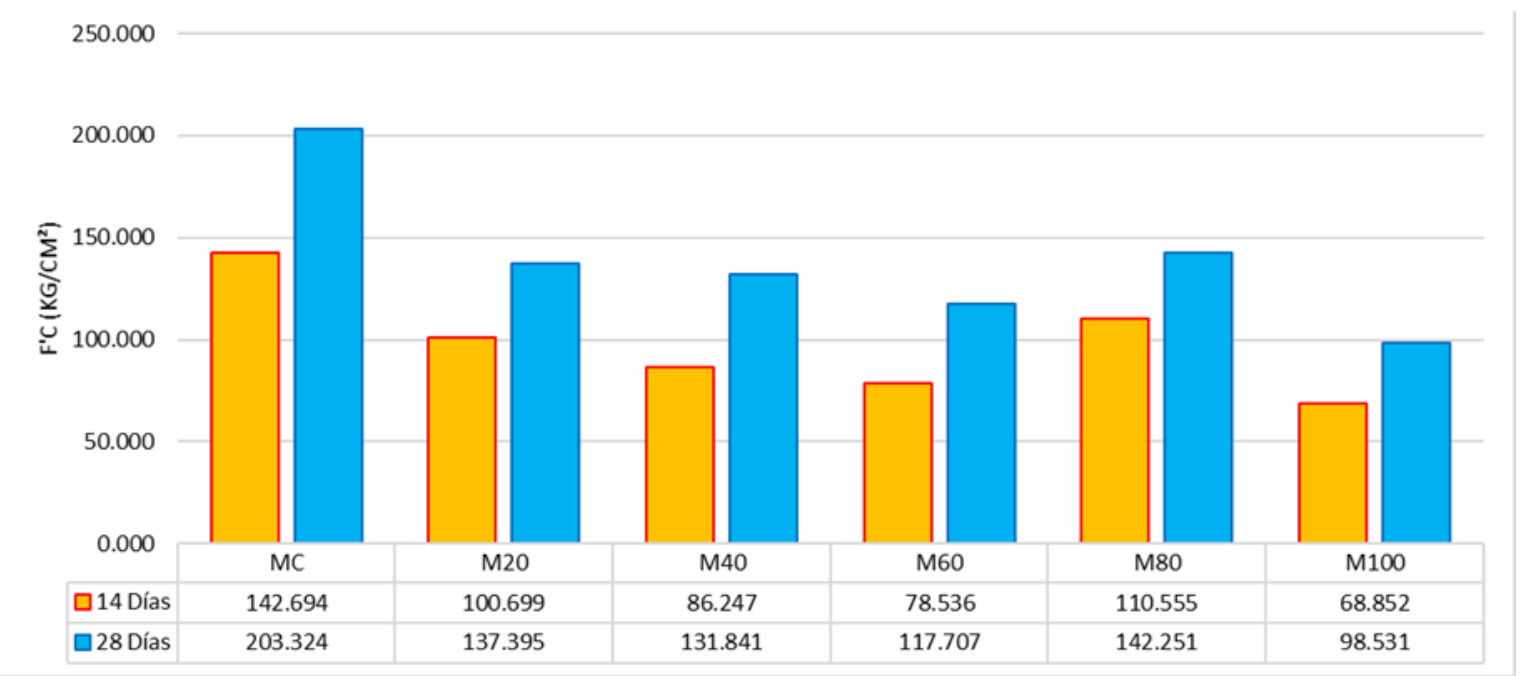

Gráfica 4.- Resistencia a la compresión de concretos sustentables.

De acuerdo con los resultados presentados en la gráfica, se aprecia que a los 14 días las mezclas tienen menor resistencia en cuanto a la mezcla control, lo que era de suponerse por las sustituciones de material cementante y reciclado. Existiendo una variación en cuanto a la mezcla M80 que presenta un comportamiento se resistencia alto para el porcentaje que tiene de agregado reciclado. También se observa como la mezcla con el $100 \%$ de agregado reciclado tiene una resistencia que alcanzo el $50 \%$ en referencia a la mezcla control. Todas las mezclas a los 28 días presentan que siguen desarrollando resistencia, pero presentando en todas también, un decremento en la resistencia en referencia con la mezcla control.

\subsubsection{Módulo de elasticidad.}

En el gráfico 5, se pueden observar los resultados de las diferentes mezclas elaboradas, a la edad de 28 días, el concreto que presento mejor desempeño fue el de sustitución de $20 \%$ de agregado reciclado, teniendo un resultado por encima del concreto normal de $12.09 \%$, la mezclas con sustitución de $60 \%$ presentó una mejora de igual manera por encima del concreto de referencia de $4.97 \%$. Los resultados obtenidos no se podrán comparar con otras investigaciones debido al método de ensayo que se utilizó fue el de dos anillos un micrómetro. Por lo cual obtenemos menos deformación y los módulos de elasticidad se elevan, estos resultados dan un parámetro del comportamiento elástico de cada concreto sustentable elaborado.

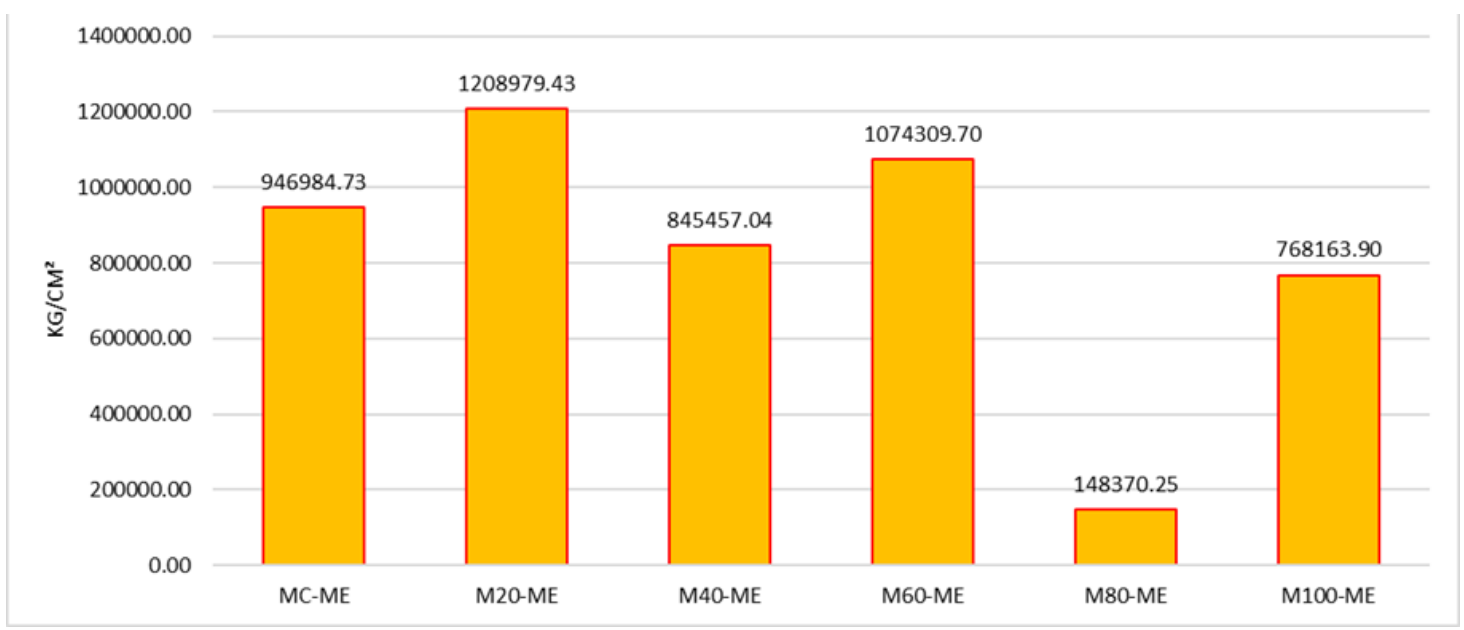

Gráfica 5.- Modulo de elasticidad de concretos sustentables. 


\section{CONCLUSIONES}

Los resultados de las temperaturas obtenidas son de acuerdo a las condiciones climáticas cuando se realizaron las mezclas de concreto sustentables ternarios. Dichos resultados están dentro de las especificaciones de la norma NMX-C155. El ensaye de revenimiento, se obtuvieron las diferentes consistencias de los concretos ternarios, en los cuales se apreció una disminución de consistencia al aumentar el porcentaje de las adiciones de agregado reciclado, esto se atribuye a que las diferentes densidades y absorciones de las adiciones. Las masas unitarias de los concretos sustentables se encuentran dentro de las especificaciones para ser utilizados como concretos de uso estructural.

En cuanto a los resultados del concreto en estado endurecido se observó que la mezcla con el $80 \%$ de $\mathrm{AR}$, presentó mejor comportamiento en cuanto a resistencia, que las otras y muy cercano a la mezcla control. Además, que en el módulo de elasticidad la mezcla con $20 \%$ presenta un mejor comportamiento, esto por tener mayor porcentaje de AN, y rebasa el valor que obtiene la mezcla control.

\section{AGRADECIMIENTOS}

M.A. Baltazar-Zamora, et. al., agradecen a PRODEP por el Apoyo otorgado por la SEP, al Cuerpo Académico UV-CA-458 "Sustentabilidad y Durabilidad de Materiales para la Infraestructura Civil", en el marco de la Convocatoria 2018 para Fortalecimiento de Cuerpos Académicos con IDCA 28593. Así también A. Landa-Sanchez, agradece al CONACYT por la beca de otorgada para cursar el programa de Doctorado en Ingeniería perteneciente a FIME-Xalapa de la Universidad Veracruzana.

\section{REFERENCIAS}

A. Landa Sánchez, A.E. Landa Gómez, J.M. Mendoza Rangel, F. Almeraya Calderón, R. Croche Belín y M.A. Baltazar Zamora (2009), Comportamiento de la corrosión del acero AISI 316 como refuerzo en Concretos Ternarios Sustentables con sustitución parcial de CPC en 10\%, $20 \%$ y 30\% de CBCA-HS inmersos en Agua de Mar. 34 ${ }^{\circ}$ Congreso Nacional de la Sociedad Mexicana de Electroquímica, 2019 y 12th Meeting of the Mexican section of the Electrochemical Society. Querétaro, México.

Aldo Emelio Landa-Gómez, René Croche, Sabino Márquez-Montero, Rafael Villegas Apaez, Ha ArizaFigueroa, Francisco Estupiñan López, Citlalli Gaona Tiburcio, Facundo Almeraya Calderón, Miguel Angel Baltazar-Zamora (2018). Corrosion Behavior 304 and 316 Stainless Steel as Reinforcement in Sustainable Concrete Based on Sugar Cane Bagasse Ash Exposed to Na2SO4. ECS Transactions Vol. 84. Pags. 179-188

Aldo Landa-Gómez, Saúl Castillo Aguilar, Jose Alberto Reyes Jimenez, Rafael Villegas Apaez, Ricardo Galván-Martínez Sr., Jose A. Cabral-Miramontes, Citlalli Gaona Tiburcio, Facundo Almeraya and Miguel Angel Baltazar-Zamora. (2018). Evaluation of the Corrosion of AISI 304 Stainless Steel Embedded in Sustainable Concrete with High Volumes of Scba-SF Exposed in Marine Environment. ECS Transactions

Aldo Emelio Landa-Gómez, René Croche, Oscar Manuel Lopez Yza, Ricardo Galván Martinez, Jose A. Cabral Miramontes, Citlalli Gaona Tiburcio, Facundo Almeraya Calderón, Miguel Angel BaltazarZamora (2018), Corrosion Behavior of AISI 316 Stainless Steel As Reinforcement in Ternary Sustainable Concrete Based on Scba-SF Exposed in Seawater. ECS Transactions

Ariza H.A., Croche R., Gaona C., Almeraya F., Ramírez A.C., Villegas R., Márquez S., Baltazar M.A. (2019), "Evaluación de la corrosión del acero galvanizado y acero AISI 304 embebidos en

concretos ecológicos con sustitución de CPC por 10, 20 y 30\% de CBCA-HS expuestos a $\mathrm{Na}_{2} \mathrm{SO}_{4}$ al 3.5\%" in Meas Y., Morales J. Frontana C.E. (Eds), Corrosión y tratamientos superficiales, SMEQ, Querétaro (México), pp. 
Baltazar-Zamora M.A., Santiago, G., Moreno V., Croche R., De la Garza M., Estupiñan, F., Zambrano, P., Gaona, C. (2016). Electrochemical Behaviour of Galvanized Steel Embedded in Concrete Exposed to Sand Contaminated with $\mathrm{NaCl}$. Int. J. Electrochem. Sci. 11, 10306-10319, doi: 10.20964/2016.12.28. De Vries, P. (1996), "Concrete recycled: crushed concrete aggregate". In: Proc. of the International Conference: Concrete in the Service of Mankind. I. Concrete for Environment Enhancement and Protection, Dundee, Scotland, pp. 121-30.

Dhir Acker Av. (1996), Recycling of concrete at a precast concrete plant. Betonwerk+Fertigteil-Tech 1996; 6:91-101.

Dhir R, Henderson N, Limbachiya M., Editors, (1998). Proceedings of International Symposium: Sustainable Construction: Use of Recycled Concrete Aggregate. Thomas Telford.

García D.L, Cabral J.A, Gaona C., Almeraya F., Ramírez A.C., Croche R., Baltazar M.A. (2019), "Influencia del medio ambiente de exposición y tipo de curado en la cinética de corrosión de concreto reforzado" in Meas Y, Frontana C. E., Pérez J. de J. (Eds), Electrodepositos y modificación de superficies, SMEQ, Querétaro (México), pp.

García D.L, J.A. Cabral M., C. Gaona-Tiburcio, F. Almeraya-Calderón, A.C. Ramírez-García, R. Villegas-Apaez, S. Marquez-Montero, M.A. Baltazar-Zamora (2019), "Eficiencia anticorrosiva del acero AISI 304 como refuerzo en concretos ecológicos ternarios base CBCA-HS expuestos a sulfato de magnesio." $34^{\circ}$ Congreso Nacional de la Sociedad Mexicana de Electroquímica, 2019 y 12th Meeting of the Mexican section of the Electrochemical Society. Universidad Veracruzana. Querétaro, México. Landa A., Gaona C., Almeraya F., Ramírez A.C., López O. M., Vélez J.A., Solorzano R., Márquez S., Baltazar M.A. (2019), "Evaluación electroquímica del acero inoxidable AISI 316 embebido en concretos sustentables base ceniza de bagazo de caña de azúcar expuestos a un medio marino" in Meas Y, Frontana C. E., Pérez J. de J. (EDS), Electrodepositos y modificación de superficies, SMEQ, Querétaro (México), pp.

Landa Gómez (2018). Evaluación de la corrosión de concretos sustentables ternarios inmersos en agua de mar. Tesis maestría. Universidad Veracruzana.

Landa A.E., Croche R., Márquez S., Galván R., Gaona C., Almeraya F., Baltazar-Zamora M.A. (2018). Correlation of Compression Resistance and Rupture Module of a Concrete of Ratio $w / c=0.50$ with the Corrosion Potential, Electrical Resistivity and Ultrasonic Pulse Speed, ECS transactions, 84, 217-227 Limbachiya MC, Leelawat T, Dhir RK., (2000), "Use of recycled concrete aggregate in high-strength concrete", Mater Struct, Vol. 33, pp. 574-580

O. Ojeda Farías, J.M. Mendoza Rangel, M. A. Baltazar Zamora (2018). Influence of sugar cane bagasse ash inclusion on compacting, CBR and unconfined compressive strength of a subgrade granular material. Alconpat internacional 8 (2): 194-208.

ONNCCE. (1997). Industria de la Construcción - Agregados - Reducción de las Muestras de Agregados Obtenidas en Campo al Tamaño Requerido para las Pruebas. México: ONNCCE.

ONNCCE. (1997). Industria de la Construcción - Agregados para Concreto - Análsisis Granulométrico - Método de Prueba. México: ONNCCE.

ONNCCE. (1997). Industria de la Construcción - Concreto - Terminología. México: ONNCCE.

ONNCCE. (2004). Industria de la Construcción - Agregados - Masa Volumétrica - Método de Prueba. México: ONNCCE.

ONNCCE. (2004). Industria de la Construcción - Agregados - Muestreo. México, MÉXICO: ONNCCE.

ONNCCE. (2004). Industria de la Construcción - Agua para Concreto - Especificaciones. México: ONNCCE.

ONNCCE. (2006). Industria de la Construcción - Agregados - Contenido de Agua por Secado - Método de prueba. México: ONNCCE. 
ONNCCE. (2010). Industria de la Construcción - Agregados - Determinación de la Resistencia a la Degradación por Abrasión e Impacto de Agregados Gruesos Usando la Máquina de los Ángeles. México: ONNCCE.

ONNCCE. (2010). Industria de la Construcción - Concreto Hidráulico - Cabeceo de Especímenes. México: ONNCCE.

ONNCCE. (2010). Industria de la Construcción - Concreto Hidráulico - Determinación de la Temperatura del Concreto Fresco. México: ONNCCE.

ONNCCE. (2010). Industria de la Construcción - Concreto Hidráulico - Determinación del Revenimiento en el Concreto Fresco. México: ONNCCE.

ONNCCE. (2013). Industria de la Construcción - Concreto Fresco - Muestreo. México: ONNCCE.

ONNCCE. (2013). Industria de la Construcción - Concreto Sometido a Compresión - Determinación del Módulo de Elasticidad Estático y Relación Poisson. México: ONNCCE.

ONNCCE. (2014). Industria de la Construcción - Agregados - Determinación de la Densidad Relativa y absorción de Agua del Agregado Fino - Método de Ensayo. México: ONNCCE.

ONNCCE. (2014). Industria de la Construcción - Agregados - Determinación de la Densidad Relativa y Absorción de Agua del Agregado Grueso. México: ONNCCE.

ONNCCE. (2014). Industria de la Construcción - Agregados para Concreto Hidraulico Especificaciones y Método de Ensayo. México: ONNCCE.

ONNCCE. (2014). Industria de la Construcción - Concreto Hidráulico - Determinación de la Masa Unitaria, Cálculo del Rendimiento y Contenido de Aire del Concreto Fresco por el Método Gravimétrico. México: ONNCCE.

ONNCCE. (2014). Industria de la Construcción - Concreto Hidráulico - Dosificado en Masa Especificaciones y Método de Prueba. México: ONNCCE.

ONNCCE. (2014). Industria de la Construcción - Concreto - Determinación de la Resistencia a la Compresión de Especímenes - Método de Ensayo. México: ONNCCE.

ONNCCE. (2016). Industria de la Construcción - Concreto - Elaboración y Curado de Especímenes de Ensayo. México: ONNCCE.

NMX-C-1690NNCCE-2009, "Extracción de especímenes cilíndricos o prismáticos de concreto hidráulico endurecido.

IMCYC (Construcción y tecnología del concreto, Enero 2015)

Ruíz M., Landa A., Cabral J.A., Gaona C., Almeraya F., Malpica R., Márquez S., Baltazar M.A. (2019), "Influencia del tipo de acero en el comportamiento de la corrosión de concretos sustentables ternarios elaborados con ceniza de bagazo de caña de azúcar y humo de sílice expuestos a sulfato de sodio" in Meas Y., Morales J. Frontana C.E. (Eds), Corrosión y tratamientos superficiales, SMEQ, Querétaro (México).

Santiago, G., Baltazar-Zamora M.A., Galván, R., López, L.D., Zapata, F., Zambrano, P., A. Gaona, C. and Almeraya, F. (2016), Electrochemical evaluation of reinforcement concrete exposed to soil type sp contaminated with sulphates. Int. J. Electrochem. Sci. 11,4850-4864, doi: 10.20964/2016.06.31

Santiago, G., Baltazar-Zamora M.A., Olguín, J., López, L.D., Galván, R., Ríos, A. Gaona, C. and Almeraya, F. (2016), "Electrochemical evaluation of a stainless steel as reinforcement in sustainable concrete exposed to chlorides". Int. J. Electrochem. Sci. 11, 2994

Zankler G., (1999)., "Recycled materials in concrete construction: fields of application, development tendencies and quality assurance”., Betonwerk+Fertigteil-Tech, Vol. 4, pp. 38-43. 